\title{
Protée
}

\section{L'ambiologie, forme contemporaine de la rumeur}

\section{Simon Harel}

Volume 32, numéro 3, hiver 2004

\section{La rumeur}

URI : https://id.erudit.org/iderudit/011255ar

DOI : https://doi.org/10.7202/011255ar

Aller au sommaire du numéro

\section{Éditeur(s)}

Département des arts et lettres - Université du Québec à Chicoutimi

\section{ISSN}

0300-3523 (imprimé)

1708-2307 (numérique)

Découvrir la revue

\section{Citer cet article}

Harel, S. (2004). L'ambiologie, forme contemporaine de la rumeur. Protée, 32(3), 9-24. https://doi.org/10.7202/011255ar

\section{Résumé de l'article}

Les thèses actuelles sur la délocalisation euphorique des communautés de sens et la virtualisation électronique des réseaux de communication appartiennent à un paradigme techniciste qui s'épuise peu à peu. L'euphorie de ces dernières années (de la croissance effrénée de l'indice boursier NASDAQ à la valorisation de "l'autoroute électronique ") cache un désarroi profond qu'il convient d'analyser. La surveillance et la répression ont laissé place à la gouvernance préventive, au gouvernement " en ligne ". L’ancienne théorie des jeux, tout comme la sémiotique sont devenues des applications industrielles dont la technicité opératoire permet de résoudre conflits et problèmes. Encore là, rien qui ne soit très surprenant. Reste une question centrale : comment penser la composition de la rumeur publique à la suite du 11 septembre, l'utilisation systématique du discours médiatique tenant lieu de relais rhétorique et sémiotique de la première importance ? Pour quiconque s'émeut aujourd'hui des rumeurs publiques qui accompagnent la dissémination " contagieuse " de cette menace qu'incarne l'étranger (celui qui est coupable de " délit de faciès " : arabe ou musulman), il y a matière à méditation.
Ce document est protégé par la loi sur le droit d'auteur. L’utilisation des services d’Érudit (y compris la reproduction) est assujettie à sa politique d'utilisation que vous pouvez consulter en ligne.

https://apropos.erudit.org/fr/usagers/politique-dutilisation/ 


\title{
L'AMBIO LO GIE, FO RM E CO NTEM PO RAIN E DE LA RU M EU R
}

\author{
SIMON HAREL
}

\section{UNE PAROLE ÉVANESCENTE}

La rumeur repose sur le pouvoir d'une parole évanescente. C'est un constat qu'il nous faut explorer avec plus de rigueur. Chaque fois qu'il est question de la rumeur, on entend par là un discours qui s'immisce, qui fait effraction, qui prend la forme troublante d'une ambiance qui paralyse le sujet ainsi interpellé. «L'objet» de la rumeur est pris au piège d'un monde qui est à la fois répétitif et évanescent. On ne peut éviter la rumeur, à moins d'être un véritable spartiate qui ignore les attaques insidieuses auxquelles il est soumis. Veut-il réagir de manière trop affirmative, le sujet sera accusé de délit d'interprétation. «Tiens, celui-là est mal à l'aise. L'accusation, d'où qu'elle vienne, ne lui plaît pas». Et le verdict tombera: "coupable».

C'est que la rumeur caractérise une situation troublante: elle suppose le maintien de la présomption d'innocence tout en infligeant une souffrance psychique, une peine morale qui circonscrit le champ d'application du oui-dire. Ainsi, la rumeur inaugure un dispositif rhétorique d'une grande efficacité. Le système juridique, on le sait, repose sur un codex: des articles de lois, une jurisprudence sont autant de sources référentielles qui imposent une délibération méthodique où le témoignage est soumis à la rigueur d'un contre-interrogatoire.

Il en va autrement de la rumeur qui a comme caractéristique première d'être volage. Elle met en relief des situations qui font prévaloir l'imprévisibilité de la parole publique. Faut-il en déduire que la rumeur fait le jeu d'accusateurs anonymes et de paranoïaques tourmentés? Qu'en est-il de la rumeur aujourd'hui? Possède-t-elle un grand pouvoir d'évocation collectif, une acidité intrusive et dévastatrice? Sommes-nous encore à l'ère de la rumeur (comme d'autres peuvent parler de "fait divers»)?

Je n'ai pas ce sentiment tant les configurations narratives qui définissent notre relation à la rumeur ont changé d'aspect de manière radicale. Bien sûr, la propagation de la rumeur est un sujet herméneutique de choix. Encore plus que le témoignage (qui repose sur l'inamovibilité de la parole assermentée), la rumeur est une praxis dont la fluidité est la forme vive. Discours ambiant, la rumeur est un phénomène retors et arbitraire qui obéit aux formes subjectives de la délation. La 
rumeur vous tombe dessus, littéralement, véritable poisse qui vous empêche de vous sentir libre. Mais l'étude de la rumeur correspond-elle en tous points au primat de l'identité narrative qui impose la création d'une signification à interpréter? Je ne le crois pas. La rumeur ne compose pas d'emblée un récit. Elle n'obéit pas à des règles factuelles qui sont posées de manière déterminée: pas de narrateur explicite, pas d'auditoire dûment identifié, pas de prévisibilité des séquences narratives.

En ce sens, la rumeur appartient au monde de la narration orale. Elle ne représente pas dans tous les cas un discours écrit. La rumeur, au contraire, suppose que le contenu raconté soit en grande partie indéterminé. Sur ces questions, la rumeur prend cependant ses distances avec un segment de la tradition orale qui, sous l'aspect du récit légendaire, a pour rôle de transmettre un contenu fondateur. Il en va autrement de la rumeur qui appartient à une économie de la délation. À cette différence que la rumeur tout comme le bavardage se plaisent à entretenir une «ambiologie» diffuse. La délation est accusatrice. C'est un discours dévoyé qui appartient à un monde hostile où le sujet est fiché, pointé du doigt, puis incarcéré. Ce sont des scénarios sombres que nous évoquons ici. La délation ne prône pas un discours diffus. Elle est au contraire sectaire et tranchante. La délation s'empare de vous corps et âme. Elle ne vous laisse pas de repos, vous enferme et vous tue.

En comparaison, la rumeur est un mal bénin dont on aurait tort de se plaindre. Ne constitue-t-elle pas une forme pérenne de la socialité? Ne correspond-elle pas à cette «solidarité organique» qui contribue, selon Émile Durkheim, à former la trame de la communauté? Si nous retenons l'hypothèse de cette solidarité organique, on peut ajouter que la rumeur est l'équivalent du Moi-peau décrit par Didier Anzieu. D'un point de vue psychanalytique, le Moi-peau est une représentation mi-psychique, mi-physiologique qui contribue à former un sentiment d'identité qui rappelle à bien des égards cette ambiologie dont je me fais le défenseur.
À s'en tenir à cette perspective, la rumeur est encore un «mal» bénin. Le bavardage, la rumeur seraient des formes artisanales de la délation. À l'encontre de cette dernière qui cible son objet (sa proie) avec violence, le bavardage et la rumeur correspondent à une logique de voisinages. Tout compte fait, bavardage et rumeur appartiennent à un monde qui met à l'avant-scène, de manière formelle, une logique identitaire. Ce n'est pas un hasard si nous faisons appel aux représentations de la solidarité organique et du Moi-peau. Dans les deux cas, il est question d'une identité et d'une vérité révélées par l'entremise du discours de l'affect corporel et de la communauté sociale. Ainsi, la rumeur suppose la présence d'un interlocuteur masqué qui assure la transmission de propos jugés secrets ou inavouables. De ce point de vue, la rumeur appartient de façon déterminante au monde du récit et privilégie la forme prolixe d'un discours oral. On pourra toujours faire valoir que la rumeur s'accommode de l'écrit. Il reste que l'énonciation de la rumeur est impersonnelle. Ainsi, la lettre anonyme est un exemple précieux de rumeur qui prend la forme d'un document et parfois d'une signature. Que le document soit véridique, que la signature soit falsifiée, tout cela importe peu en regard du rôle que joue la rumeur. Cette dernière incarne une ambiologie de culture orale qui fait prévaloir la transmission invérifiable de propos dits secrets.

Prenons le motif de la rumeur publique qui nourrit conversations et allusions à propos de l'univers de la prostitution juvénile à Québec. La presse électronique et écrite ne peut se permettre de franchir ce cap où la rumeur devient fait avéré. Le système juridique est là pour protéger le citoyen, pour assurer sans entraves la jouissance de son droit à la vie privée. On ne défend pas sa «réputation» sans raison! Cette dernière est l'émanation la plus claire de ce que la rumeur altère sans relâche. Que veut dire en effet défendre sa réputation, sinon tenter de préserver une image de marque? Cette dernière n'est-elle pas l'incarnation d'un ethos publicitaire dont le sujet fait sans relâche la réclame? Qu'en est-il alors de la 
promotion du droit à la vie privée, à la réputation: figure qui concorde avec la position dominante d'une logique identitaire?

\section{FICTION ET VÉRITÉ: LES MUTATIONS DE LA RUMEUR}

De façon significative, la rumeur est devenue un élément constitutif de la publicisation postmoderne de l'image de marque. Plusieurs facteurs peuvent être évoqués dont la forme descriptive est néanmoins l'indication d'une mutation de la «rumeur».

L'évolution récente du discours littéraire correspond à cette tendance. Il y a peu, le journal intime, les mémoires, sans compter l'autobiographie, constituaient les formes discursives d'une identité «pure». On se plaisait à opposer au discours romanesque (indicateur exemplaire de fictivité) l'existence de discours plus vrais que nature qui caractérisaient la mise en jeu d'un référent indiscutable. Bien sûr, la comparaison est sommaire. Elle a pour seul mérite d'indiquer que nos indices de fictivité bougent de manière considérable. Jusqu'à tout récemment, on lisait l'autobiographie comme l'exemple d'un discours à forte connotation de référentialité. Ainsi, la lecture de L'Âge d'homme nous plongeait au cœur d'une subjectivité qui octroyait une large place à l'inconscient, aux confessions personnelles, aux révélations sulfureuses. En somme, on lisait Leiris comme autrefois Rousseau, avec le souci de mieux connaître la "profondeur» subjective de l'écrivain, le registre personnel de son roman familial, de son enfance, puis de son âge d'homme.

Dans cette perspective, la rumeur perdait de sa valeur intrinsèque. L'autobiographie, telle que la pratiquait un Leiris, devenait une façon de désavouer le caractère diffus du discours public que représentait traditionnellement la rumeur. Quelle utilité y avait-il à colporter ragots et insinuations sur la vie "privée" d'un auteur alors que ce dernier ne se gênait pas pour révéler les détails les plus personnels de sa sexualité et de sa vie amoureuse? La rumeur s'en trouvait courtcircuitée. Alors que le roman était l'aveu d'un fort indice de fictivité (par l'identification imaginaire du sujet lecteur aux scénarios narratifs mis en place), l'autobiographie se caractérisait par son littéralisme. C'est ainsi que l'autobiographie, forme documentaire de la conscience de soi, connut ses heures de gloire. Alors même que le Nouveau Roman apparaissait, l'autobiographie expérimentale d'un Leiris abandonnait peu à peu le modèle confessionnel (de L'Âge d'homme) pour les perspectives plus nouvelles de l'association libre et de l'interprétation psychanalytique.

Des documents ethnographiques de première main à la génétique du texte, le discours théorique contemporain manifestait un vif intérêt pour les formes diverses de la culture matérielle. Ce projet concordait avec la revalorisation du discours populaire et des lieux d'énonciation des sujets subalternes. Dans ce discours critique, l'indice de fictivité était encore une fois minimal. Par l'historiographie de la culture populaire, il était possible de mettre un terme au discours autoritaire de la rumeur publique. Par la prise en compte des énonciateurs anonymes, des outsiders, des marginaux, la parole était offerte à des sujets autrefois relégués aux marges de l'histoire officielle. Alors que la rumeur incarnait le discours diffus des manants et des prolétaires, une modification substantielle du statut de la rumeur indiquait une transformation des paradigmes d'interprétation. Dans le contexte de l'histoire récente du Québec, le documentaire (ou le cinéma direct) traduisait la revendication d'une culture matérielle vivante et inventive.

En histoire, ethnologie urbaine et sociologie de la culture, cette école de pensée (de Pierre Sansot à Michel de Certeau) combattit avec force l'idéologie de la rumeur qui était perçue comme la manifestation d'une pensée élitiste. La rumeur, pour ces théoriciens, traduisait un mépris sans bornes pour les tenants d'une culture populaire dynamique. De leur côté, les théoriciens de la génétique du texte, dans la foulée de l'école sociocritique, étudiaient des documents dont la fonction paratextuelle était autrefois négligée. Pour les généticiens du texte, la rumeur scripturaire (le paratexte) avait autant d'importance que l'objet esthétique. Le livre ne représentait plus un monument littéraire dont l'immatérialité historique 
(l'atemporalité) ne pouvait être remise en question. Complément de cette modification de la rumeur, cette dernière perdait sa puissance évocatrice. Le manuscrit, ce n'était plus seulement le témoignage d'un labeur, d'une inspiration: formes imparfaites et artisanales d'une intention créatrice. Le manuscrit devenait un référent digne de foi dans cette quête d'une technè organisatrice de sens. Il incarnait une nouvelle rationalité qu'il convenait d'intégrer à nos formes contemporaines d'investigation scientifique.

Que ce soit la parole populaire (des prolétaires), le discours des sujets exclus (femmes, enfants) ou le paratexte littéraire, l'enjeu était de rendre justice à la rumeur (en reconnaître la pertinence) tout en l'insérant dans un cadre formel d'analyse. La volonté de donner la parole à l'informateur (anthropologique), aux mouvements autrefois marginaux (de la défense des droits des femmes à ceux des enfants), était riche en conséquences. Fait majeur, l'écriture de l'histoire proposait une anthropologie respectueuse des sans-voix et des sanslogis. En somme, le discours théorique avait pour fonction de contrecarrer une perception romantique de la rumeur.

\section{UN TOHU-BOHU}

À l'entendre dans son acception traditionnelle, la rumeur est en effet un discours diffus, un tohu-bohu: diverses qualifications qui ont pour fonctions d'indiquer que la rumeur est lointaine et ne possède pas d'énonciateur autorisé. De la rumeur d'Orléans (Edgar Morin) aux discours actuels sur les ramifications du terrorisme cybernétique, cette idée d'une "parole» volatile et impure prévaut. Dans les univers de l'espace propre (de l'école à la prison, du réseau hospitalier à l'hospice), la rumeur n'existe pas ou si peu. Elle est toujours située hors les murs, comme s'il fallait qu'une forteresse écotopique atténue son caractère troublant, ensorcelant. À l'encontre du caractère délibératif de l'agora, la rumeur traduit un discours paradoxal. Elle se situe "en marge» de l'histoire officielle, à l'instar de la parole des prolétaires et des sans-logis. Mais le statut périphérique de la rumeur n'est pas fait que d'inconvénients. La rumeur est aussi le fantasme d'une parole vivante. L'énonciateur de la rumeur offre la promesse d'une autre Loi qui mettrait un terme aux avanies anciennes.

Dans la crainte qu'inspire, par exemple, la rumeur politique, il y a cette idée qu'un contre-discours existe, même si sa localisation n'est pas déterminée. Ces deux formes, apparemment antagonistes, font de la rumeur un discours prolixe. La parole des prolétaires est «à peine» un discours que ne veulent pas entendre, encore moins reconnaitre, les institutions du pouvoir. La rumeur, parce qu'elle échappe à la technè politicienne, semble un discours spontané. Mais la rumeur, heureusement, ne se réduit pas au fantasme d'une parole sans contrainte qui nous amènerait à y voir l'idéal rousseauiste, en tous les cas utopique, d'un monde naturel qui échappe aux contraintes de la délibération politique. Il y a beaucoup de naïveté dans ce dernier discours. C'est ainsi, dit-on, que la rumeur précède l'histoire. Elle incarne une rationalité dont la compréhension n'est pas encore réalisée dans un «ordre du discours». Dans cette perspective, la rumeur est une "voix», une présence.

À cette parole vivante, parfois retardataire, parfois émancipatrice, il faut cependant opposer une autre définition de la rumeur qui définit un système entropique, sans aucune propriété énonciative personnelle. La rumeur, ce ne serait plus la prise de parole des mouvements révolutionnaires et prolétaires. Sous sa forme contemporaine, elle incarnerait un bruit de fond insistant qui ne possède plus de valeur sémantique. Cette définition de la rumeur caractérise, à mon sens, les formes caricaturales du discours culturel contemporain. Le désir de recycler les formes architecturales du passé (du néovictorien à l'Arts and Craft) fait de la rumeur un signe à indexer au rang de nos dictionnaires référentiels.

On opposera que la rumeur n'est pas un signe, qu'on ne peut en définir la forme. Pourtant la rumeur est bien présente dans la mise au jour de nos mythologies contemporaines. On redoute la rumeur qui vous donne le sentiment de ne plus être actuel. 
Elle vous dit que le néovictorien n'est plus à la mode, que votre code vestimentaire est désuet, que votre nouvel agenda électronique est déjà obsolète. Nos références ambiologiques contemporaines sont précaires, tant les anciennes «mythologies» barthésiennes cèdent le pas devant le constat d'une inactualité du monde contemporain.

Ce constat a été amplement développé depuis une vingtaine d'années: l'ère du vide, de l'indifférence, de la passion narcissique a été inventoriée à l'excès. Notre discours théorique privilégie toujours ce point de vue négatif qui dit l'absence de communication, de signification, de réalité. À prendre pour exemple les communautés urbaines (de banlieues) qui se regroupent par affinités d'âge, de statut social, de projets "de vie», la rumeur est l'indication effarante d'un «vous n'existez plus», "vous n'êtes plus conforme aux représentations de ce qui est "vivant" ". On trouvera peut-être l'expression excessive. Pourtant, la rumeur traite de ce droit de vie et de mort. Autrefois, la rumeur provenait des faubourgs des quartiers ouvriers, des repaires d'insurgés. Elle faisait référence, de façon diffuse, à la menace que représentaient des communautés d'exploités que l'on tentait, avec difficultés, de maintenir à distance. La rumeur actuelle est ambiologique, publicitaire. Elle ne correspond plus aux critères de centralité et de périphérie qui animent nos représentations spatiales. Le citoyen d'une communauté «modèle» peut bien recréer, à quarante ou soixante kilomètres d'Atlanta, l'idéal d'un mode de vie "contemporain"; il reste que la rumeur est un acide corrosif qui condamne à la dégradation rapide toute forme de socialité.

Un jour, cette banlieue ne sera plus celle où il faut habiter. L'échangeur routier, si rapide, qui permet de se déplacer uptown ou downtown sera congestionné. Les services publics se dégraderont, de leur côté, par suite d'une taxation minimale et du faible pouvoir de représentation civique des institutions municipales. Des populations «indésirables» (nouveaux immigrants à la recherche d'une "place» dans le tissu social, habitants de quartiers limitrophes plus pauvres) contribueront à modifier ce que fut, pour un temps, l'«image de marque» d'une communauté modèle. Cette description banale de la déshérence d'un quartier est un «lieu commun» des études urbaines. De Saint-Louis au South Bronx, nous savons, comme on dit, que les villes vivent et meurent. Dans la formulation de ce constat, les représentations spatiales ont leur importance. Mais les images de centralité et de périphérie se transforment peu à peu tant l'ambiologie (forme actuelle de la rumeur) se construit selon d'autres modes de figuration. Si la rumeur décrivait autrefois un monde représenté comme étranger, l'ambiologie fait valoir une stratégie générique de dissolution de l'identité dans son rapport singulier à un «emplacement».

Revenons à cette figure banale de l'habitant d'une banlieue d'une métropole nord-américaine. La banlieue n'est même plus ici le signe de la périphérie. À vrai dire, l'urban sprawl (le développement urbain anarchique) accentue le sentiment d'une délocalisation du lieu. Dans le domaine des études culturelles, la référence aux Nodes et autres Internet Protocols met en valeur la représentation d'un monde rhizomatique. Il suffit de lire un ouvrage sur le Los Angeles postindustriel, image d'Épinal des propagandistes de la délocalisation, pour constater que l'illusion de la virtualité (comme nouvelle forme de représentation des rapports spatiaux) est dominante. La ville, c'est le Web: les artères urbaines sont la concrétisation (toujours imparfaite et impure) de la libre circulation de «données" cybernétiques. À s'en tenir à ce point de vue, la modification de l'arsenal rhétorique qui nous permet de nommer la ville n'a fait que changer de cadre sémantique. Autrefois, l'urban sprawl, c'était le rejet d'une écologie urbaine respectueuse des milieux de vie. Ce développement anarchique était comparé à la prolifération d'un cancer: métastases et tumeurs composaient le portrait d'une organicité laissée à ellemême. En l'absence de frontières, d'espaces propres, de localisations écotopiques, la ville était comparée à une «jungle» urbaine, à un arasement de l'écosystème. La rumeur prenait la forme d'une industrialisation débridée, d'un emballement de la consommation. La 
rumeur industrielle (de l'économie tertiaire de l'aprèsguerre) répétait, à grands traits, cette figure d'une altérité diffuse et bruyante.

\section{L'IDENTITÉ-TERMINALE}

Il en va autrement aujourd'hui. Les mots clés No Exit, No Entrance, Delete sont les nouveaux indices de la rumeur technicienne. Que veulent dire en effet les Must Exit et les No Way Out du postmodernisme vieillissant? Ces énoncés privilégient l'idée d'une identité-terminale. Nous devons tirer les justes conclusions de cette asémantisation du langage. Le No Exit, substantif mortifère, est un éloge sans vergogne de la disparition. Les junkies des quartiers déshérités y trouvent leur compte tant le trépas est leur avenir. Mais nous ne pouvons nous satisfaire d'un tel éloge de la mort qui se satisfait de la certitude d'une défaite anticipée. L'identité-terminale est un acte de survie dont la forme énigmatique appartient aux rescapés, aux survivants, aux polytraumatisés de l'existence: ceux qui n'ont pas d'autres mots que No Exit, No Way Out pour dire une ancienne vie "morte", et une nouvelle vie qu'ils se contentent d'arpenter sans illusion inutile. L'identité-terminale consiste à être allé au «bout de la route». C'est une pérégrination banale que celle des survivants du monde de l'infra-ordinaire.

L'axiome de la déterritorialisation généralisée est à l'ordre du jour. Mais de façon étonnante, ce discours, qu'on lisait autrefois sous la plume d'un Deleuze, d'un Baudrillard, s'est matérialisé concrètement. Il faut oser dire que ce discours s'est matérialisé grâce à une volonté de puissance qui fait de l'économie un étalon du réel, une puissance organisatrice du monde dans lequel nous vivons. La déterritorialisation n'est plus un opérateur philosophique. Elle est, au contraire, un opérateur économique de la plus haute importance. Il suffit de constater que ce discours de la déterritorialisation rejoint à bien des égards la forme violente de la délocalisation industrielle. Il n'est pas impossible que les grandes transnationales aient maîtrisé très vite, dans la mise en jeu géopolitique de leurs arsenaux industriels, l'agencement rhizomatique que prônait Deleuze.
Le propos pourra surprendre. Quoi! la pensée de l'Anti-CEdipe aurait été instrumentalisée par le cosmopolitisme transnational des créateurs d'images: ces Gap et Benneton qui offrent la vision planétaire d'un monde pluriel. Le devenir-nomade ne serait pas autre chose que le flux des capitaux boursiers qui font fi des lois de la territorialité? Véritable inconscient machinique, le flux boursier serait devenu l'image d'un lieu ouvert à toutes les turbulences dans la mesure où le capital ne respecte rien, s'immisce dans les économies émergentes pour détruire l'oikonomos des sociétés «locales». Il n'est pas sûr que le devenirnomade du monde soit encore un projet qui correspond à nos soifs de libération. Si les flux du capital, de l'information médiatique, la banalisation du savoir intellectuel sous l'aspect d'un ready-made aisément dupliqué sont nos réalités, que faut-il en conclure? Doit-on accepter l'impossibilité de tout projet de subversion qui tenterait d'associer l'oikonomos au lieu habité?

\section{LA CONTENANCE AMBIOLOGIQUE}

À la représentation organique de la ville comme métastase envahissante, il faut ajouter que le Web représente aujourd'hui une «toile» ambiologique dont la rumeur virtuelle est l'élément décisif. Je faisais référence un peu plus tôt à la création de communautés urbaines qui prennent l'aspect de «villages» protégés, de regroupements par affinités professionnelles, économiques, sociosexuelles. La chose n'est pas nouvelle. Seule l'ambiologie - rumeur médiatique persistante - permet de comprendre que ces communautés apparaissent, puis disparaissent au croisement d'un échangeur routier, d'une bretelle d'autoroute, d'un Power Center. Dans cette logique d'appartenance au monde marchand, qui recourt à la valorisation de la sphère "récréotouristique», il importe de créer des identitéshub, ce que j'appelle des identités-terminales.

La rumeur ambiologique contemporaine est un discours d'asservissement et d'exclusion. À cette nuance près que le sujet de l'exclusion habite un espace «malade», atrophié, dont le «lieu d'être» est 
désavoué. Nous avons le sentiment trompeur que nos représentations contemporaines de la rumeur diffèrent sensiblement des discours plus anciens qui mettaient l'accent sur les distinctions du centre et de la périphérie, du pouvoir politique et des quartiers ouvriers. Ce sentiment est en partie justifié tant les représentations spatiales semblent céder le pas à un discours ambiologique diffus. Le consommateur enclavé dans sa communauté urbaine élective est un sujet contraint par l'idéologie du self-containment.

Le cocooning représente à première vue une stratégie intimiste. Mais les adeptes du cocooning sont eux-mêmes des navigateurs sur le Web, des fanatiques du cinéma maison et de l'interconnexion. L'intimité conviviale qu'ils revendiquent est technique et objectale. Elle est une niche éthologique qui protège le sujet, le relie au monde intermédiatique, sous une forme soft et altérée. À constater ce mouvement, qui gagne en popularité dans les banlieues des métropoles nord-américaines (aussi bien dire à l'échelle mondiale), il faut constater un malaise profond. L'époque présente propose un monde sans affect réel qui met en scène une sensorialité perceptive ambiante. On trouvera l'expression étrange: comment peut-on opposer avec tant de facilité le «percept» et l' «affect», la sensorialité brute et le sens diffus dont témoigne l'affect?

Le percept fait référence à une sensation violente qui voit le jour dans des situations de grave précarité psychique. Dans l'autisme et la psychose, le sujet est envahi par une sensorialité qui s'impose de manière absolue. Pensons aux écrits du président Schreber pour mieux comprendre la violence de cette intrusion perceptive qui interdit littéralement de penser. Dans ces situations, le référent a laissé place insidieusement à un signifiant évidé, forme anobjectale de signification. En somme, le référent est entrevu comme un "percept» qui n'a plus sa place à l'intérieur d'un système organisé, qu'il s'agisse de la langue, de l'économie, ou des règles de parenté. Le "percept", à vrai dire, n'est pas fonctionnel. Il est tout au plus l'indice d'une intensité sensorielle massive. Le «percept» est en somme l'équivalent de ce que le psychanalyste Wilfred Ruprecht Bion nommait, à propos des configurations psychotiques, l'«attaque contre le lien». Celle-ci se caractérise par l'exacerbation du monde des sens, à l'encontre de toute différenciation cognitive et psychique. Ainsi, la mise en retrait du référent est un phénomène majeur tant la puissance du simulacre - forme délocalisée de la rumeur - traduirait l'émoussement de la communication dans la sphère publique. Cet émoussement des aspérités langagières de la communication (de la scène de ménage à la délibération politique) est la forme implicite d'une plongée «socialisée» dans le monde de la psychose.

Qu'on me comprenne bien: je n'adopte pas sur ces questions un point de vue moraliste, tout comme je ne désire pas faire preuve de défaitisme ou de cynisme. Ce retrait affectif me semble offrir un portrait assez probant de l'état des lieux de la culture contemporaine. Parler de psychose ne va pas sans généralisations dont il faut se garder. Je ne dis pas que le monde contemporain est psychotique, façon de dire qu'il est «anormal». J'avance tout au plus que le retrait affectif caractérise le monde de la délocalisation virtuelle. Dans cette perspective, le signifiant ne vaut plus pour un autre signifiant, selon le principe d'une hiérarchisation linguistique. De son côté, le «simulacre» n'a rien en commun avec l'économie de l'arbitraire du signe. Il est au contraire la forme même de la rumeur contemporaine, en ce qu'elle engage une réflexion sur la désensibilisation de nos lieux habités. Cela veut dire que le retrait affectif ou la dissociation progressive de «l'appareil à penser les pensées» (Bion) sont les témoignages probants de ruptures des formes anciennes de l'habitabilité.

\section{UN AFFECT ÉMOUSSÉ}

De façon singulière, le cocooning, la délocalisation virtuelle du Pay Per View (la commande de films «à la demande" par l'entremise des réseaux de câblodistribution) sont les indices d'un monde qui fait de la rumeur marchande l'une des formes actuelles de l'intimité et de l'habitabilité. Faire référence à cette plongée «socialisée» dans le monde de la psychose ne 
veut pas dire qu'il faut reprendre le pathos rhétorique des "fins du monde» et des fins de l'histoire. Je ne crois pas que nous gagnions à tenir ce discours qui fait intervenir la forme dite pathologique comme norme d'interprétation du discours social. Je ne revendique pas un devenir-psychotique du monde! De la même manière qu'Alain Mons parle de "plongée paysagère" afin de décrire le Zabriskie Point d'Antonioni - et la façon dont ce dernier filme le panorama de la Death Valley -, il faut entendre, dans ma référence au retrait affectif, une immersion douloureuse et océanique dans le monde énigmatique de l'ambiologie.

Le discours social actuel nous donne l'impression d'une «mobilité extrême» comme si le nouveau sujet des réseautages électroniques était plus que jamais libre de circuler à sa guise. Ce réseautage est en grande partie fictif: il correspond à une sémiotique de l'impartition dont la seule logique est de répéter à loisir les formes minimales de l'insourcing et de l'outsourcing. À la suite des travaux de Jean Baudrillard sur l'économie politique du signe qui mettaient l'accent sur l'«emplacement» idéologique du signe (à l'encontre d'une étude linguistique ou sémiotique), il est nécessaire de réévaluer la signification de la rumeur.

À première vue, l'ambiologie est atmosphérique. Mais c'est un discours superficiel que nous tenons alors. Sous son apparence anodine, l'ambiologie est encerclante et étanche. Par l'exercice de la rumeur, nous ne cessons de créer dans les bordures de cette douleur qui nous impose de médire sur l'autre. Nous ne cessons de créer, avec cette douleur, de la médisance qui nous permet d'affirmer notre identité souveraine. On laisse trop souvent entendre que l'ambiologie est affaire de conformisme, de neutralité, d'indifférenciation. Je tiens moi-même ce discours en faisant valoir que l'émoussement de l'affect est une forme active des dispositifs ambiologiques: la figure du cocooning tenant lieu d'exemple probant. Mais la psychose (ou ce que nous nous représentons comme la psychose) est aussi le signe d'une violence implacable. Il y a, dans la psychose, douleur et cruauté. Et dans l'apparente quiétude de l'ambiologie, il y a une détresse incommensurable. La reconnaissance de cette douleur est sans doute ce qui nous effraie le plus. On a tendance à oublier que la dissolution du sens, la mise en valeur du "percept» comme seul arbitre de la réalité ne sont pas des phénomènes neutres. $\mathrm{Si}$ beaucoup craignent la cruauté littéraire d'Artaud, la rage d'un Rimbaud, exemples de vies consumées, d'exigences impérieuses à (sur)vivre envers et contre tous, reste alors la figure du cocooning: bel exemple de mort lente sur fond de conformisme social. La création de ces paravents identitaires met à distance la cruauté et la mort au profit d'une illusion tenace de vie bien tempérée.

Si le devenir-nomade du monde est une supercherie, faut-il en conclure à la fin de toute utopie, dans la mesure où celle-ci aurait été cannibalisée par la forme guerrière du capital transnational? Je ne crois pas qu'il convienne d'être si pessimiste. On peut penser au propos désabusé de Baudrillard qui fait de la «simulation de la simulation" un état du monde contemporain. Mon point de vue est différent. Dans la violence feutrée de la rumeur publique, je reconnais la tache aveugle du trauma. Ce dernier se donne à voir comme «percept» sans référent, trace fugitive du signe. Prédomine la vision anxieuse ou fébrile d'un monde qui ne fait plus sens ou qui fait sens de manière fugace et aléatoire. La «simulation de la simulation", c'est la valorisation sémiotique d'un principe d'épuisement qui affirme la nullité du référent. À suivre ce point de vue, le sens, parce qu'il fait l'objet d'une duplication, revendique haut et fort son statut de faux-semblant. Dans cette perspective, le discours ne met plus en relief la fameuse tripartition du signe qui privilégie la distinction entre signifiant, signifié et référent. Le faux-semblant attaque le domaine de la référence, tente d'invalider nos univers de discours, de nous démettre de toute responsabilité.

Je continue de mon côté à penser qu'il est possible d'imaginer des stratégies de résistance à l'égard de cet émoussement du référent et de l'affect sensible sans que nos discours prennent la forme confortable du repli sur soi. Il est nécessaire d'identifier ces lieux de résistance divers et foisonnants qui appartiennent à 
notre monde actuel. Cette tâche nous permet d'identifier des champs de force où nous interrogeons la fracture du sens, sa mise sous tension, et non pas le piètre aveu de sa dissolution. Les discours du simulacre (on pensera encore à l'œuvre de Baudrillard) ont remplacé depuis longtemps les vertus curatives de la mimesis. Avec cette dernière, nous savions que le monde était un objet de représentation. En somme, nous savions que la configuration du récit permettait de construire la réalité. Il en va autrement du simulacre qui, de façon brutale, est un véritable déni de la réalité. Le simulacre ne vise pas autre chose que la dissolution de la relation entre le signe et le référent. Alors que l'identification de lieux de résistance, au cœur de la construction de nos dispositifs écotopiques, permet de situer la provenance de la rumeur.

\section{TECHNĖ ET RUMEUR}

Cette dernière convoque un imaginaire fort ancien dont l'urbanité est un trait dominant. Dans cette perspective, la rumeur appartient au monde désorganisé de la périphérie. Les menaces de soulèvement et de révolte (en Irak et en Afghanistan) proviennent encore aujourd'hui d'espaces qui sont perçus comme limitrophes. Constat brutal, le pouvoir administratif et politique ne tolère pas la rumeur en son sein. Celle-ci ne fonde pas tant une logique de l'identité narrative (dans sa relation à la temporalité) qu'un discours spatial coercitif. Si la rumeur "circule», «se déplace», c'est qu'elle traverse des espaces contraints, des espaces qui ne cohabitent pas. Elle est la figure exacerbée d'une exclusion dont l'ambiologie est aussi la forme actuelle.

Que veut dire le nouveau discours sémiotique de la rumeur: une technè de gestion à moindre coût qui fait de la délocalisation un argument économique de taille. C'est de cela qu'il faut convenir dans notre réflexion sur l'ambiologie contemporaine. Le sujet enclavé dans sa banlieue nord-américaine est sous contrainte. L'ancienne figure de la rumeur publique ne l'atteint même plus. Elle est «numérisée», au sens où nous définissons un signal dont la capacité de «gestion» du désordre environnant est minimale. Outsourcing et Insourcing composent un portrait d'une banalité inquiétante. Que ce soit dans le confort relatif d'une chambre d'hôtel (avec la consultation des chaînes de films Pay Per View) ou au domicile, par la réception automatique d'informations électroniques (du Devoir au Electronic Frontier Foundation), grâce au courriel, le sujet contemporain est «en phase» avec l'actualité. On conviendra que la consultation du fil de presse du Electronic Frontier Foundation est plus subversive que la réception des informations financières de Bloomberg! Mais ce n'est pas l'essentiel. Dans le monde de l'hyper-information, la rumeur publique est morte. Elle laisse place à la fausse actualité d'une information en sous-traitance. Il faut prendre au sérieux les discours altermondialistes qui mettent en relief la violence des échanges économiques actuels.

Le discours de la délocalisation virtuelle fait l'impasse sur cette contingence. Les "empires" délocalisent leurs conflits et leurs inégalités. Et la figure du mondialisme, invention médiatique des années dot com, exploite sans vergogne un vieux fonds de commerce colonial. Ainsi, le président des ÉtatsUnis, Georges W. Bush, déclarait que l'outsourcing était créateur d'emplois, qu'il fallait y voir non pas une faiblesse de l'économie américaine, mais au contraire une preuve supplémentaire de sa grande faculté d'adaptation. De façon complémentaire, nos constructions théoriques reprennent, sans grande nuance, ce discours de l'outsourcing. Nous valorisons le simulacre, la simulation, la délocalisation. À vrai dire, l'idée d'une transparence de la postmodernité repose sur la duplication, forme de notre pathos actuel. Georg Simmel faisait valoir en son temps la tragédie de la culture. Walter Benjamin revendiquait la perte de l'aura au cœur du monde effervescent de la modernité industrielle. Notre condition présente donne l'image d'un pathos asignifiant. Pour le dire crûment, quelle est véritablement la différence critique entre le discours économique de l'outsourcing et nos discours théoriques sur la transparence du simulacre? À mon sens, la distinction est ténue. Nous nous voulons 
sujets d'un ethos postmoderne. Nous désavouons notre condition de sujets affectifs et sensibles.

Ainsi, notre langage technique est parsemé de références au caractère corruptible du monde informatique. Norton Utilities est devenu un nouveau pont-levis qui permet de mettre à distance la menace foisonnante d'une occupation externe. Quant au corps, dans cette histoire, il en est à peine fait mention. Pourtant, le corps ne saurait tenir lieu de forme restreinte, amputée de notre relation au monde. Il est vrai que la référence à un imaginaire corporel des lieux nous plonge dans l'embarras. Le corps a déserté la maison du sens. Il est tout au plus un fragment de réel, un affect émoussé. Ce point de vue est désespérant: le corps n'est pas que déchet, flux, intensité libidinale. Au contraire, le corps est aujourd'hui l'objet d'une mise en demeure. Il nous impose d' "exister", de situer, par force repères et lieuxdits, la localisation de nos espaces habités.

Nous n'avons jamais été aussi seuls. Paradoxe troublant, nous n'avons jamais été aussi informés, «branchés» au forum vivant de l'œkoumène. Dans cette soif de partage, de mise en commun du tohubohu du monde, nous prétendons être informés de ce qui a lieu ailleurs. Mais cette connaissance est éphémère. Elle repose pour l'essentiel sur un malentendu. C'est à l'abri du monde que nous sommes informés. C'est dans la quiétude des abris du self-containment que nous prétendons tout savoir de la rumeur du monde. Alors que l'identité-terminale traduit à mon sens avec beaucoup plus de rigueur le caractère douloureux et traumatique de nos pérégrinations.

L'identité-terminale, c'est par exemple le On the Road kerouackien, ce sont aussi les pérégrinations terminales du frère malade et amnésique de Volkswagen blues, les éructations d'un Denis Vanier dans Porter plainte au criminel. L'identité-terminale, c'est un voyage qui se termine souvent par la mort ou la folie. C'est aussi, faut-il le souligner, la violence des déplacements de populations: migrations économiques (des régions déshéritées du monde aux nouveaux Eldorados), vagues de réfugiés qui tentent de sauver leur peau en négociant des passe-droits aux frontières. Dans tous les cas, l'identité-terminale met au jour les petites et grandes violences de la destitution subjective. Il y a cependant un risque réel à tenir ce discours tant nous ne pouvons confondre à loisir les déplacements à l'aveuglette de populations en quête d'un lieu de survie et le confort de l'indifférence du cocooning nord-américain.

Je suis conscient de l'impasse que produirait un tel amalgame. À voir les choses de cette manière, l'identitéterminale caractériserait un nouvel universalisme de la migration et du désespoir. Nous ne pouvons adopter un tel point de vue, tant il contribuerait à légitimer une nouvelle métaphysique identitaire des sujets déplacés. Il n'y a rien de commun entre les faux «expatriés» du jet set cybermigrant et les populations «déplacées» aux frontières de la République démocratique du Congo. Une telle précision est-elle de mise? Il le semble, tant nos discours laissent entendre, de manière confuse, que la migration est un phénomène d'époque. C'est oublier que l'identitéterminale du cocooning n'engage qu'une destitution subjective dont les conséquences sont mineures. Alors que les exodes de réfugiés traduisent une réalité bien prosaïque où l'injonction de vivre - ou de mourir - est sans cesse réitérée.

\section{LA GOUVERNANCE DE LA RUMEUR}

Il importe de noter que la rumeur n'est pas toujours polyvalente. Elle est souvent unidirectionnelle dans la mesure où ce sont les appareils de pouvoir qui en assurent la gestion quotidienne. De la République démocratique du Congo, nous savons peu. Même la rumeur n'arrive pas à percer le mur du silence. Par l'entremise de la presse spécialisée, des informations transmises de vive voix, des associations humanitaires, il est possible de savoir «à peu près» ce qui s'y passe. Mais nous sommes fort éloignés, dans ces situations d'extrême précarité, de ce flux d'informations continues que représente la rumeur. Cette dernière correspond à une masse sonore qui fait effraction de toutes parts. Nous avons une fâcheuse tendance à faire référence à la rumeur comme s'il s'agissait d'un discours volatile. Mais cette "agilité» de la rumeur est 
une illusion tenace. Le discours de la rumeur est d'autant plus fébrile qu'il se joue en des lieux connus et inventoriés.

Il est plus facile de saisir les dernières frasques de Britney Spears que de connaître les exactions commises en République démocratique du Congo. Il importe alors de redéfinir les conditions de cette ambiologie sécuritaire qui caractérise notre temps présent. Sous sa forme «anecdotique», le cocooning donne le sentiment d'un univers aseptisé. Qui pourrait s'inquiéter d'une réalité aussi banale? Pourtant, l'identité-terminale nous renvoie à notre condition de survivant. Ce n'est pas de ma part un exercice de vantardise lyrique qui serait insupportable compte tenu du propos. Être survivant n'est pas donné à tous. Ce n'est pas un choix que l'on fait. C'est une condition violente qui s'impose. L'identitéterminale est violente et traumatique. Elle fait de l'espace un territoire morcelé. Elle fait du monde psychique un univers dévasté. L'identité-terminale impose que l'on s'interroge à tout moment sur le sens de nos pérégrinations.

L'adepte du cocooning ne marche plus. Il est affalé dans une posture de mort anticipée. En fait, il ingère la rumeur, il l'incorpore de la même manière qu'il se nourrit comme un enfant que l'on gave. L'adepte du cocooning n'est jamais repu. Il appartient à une société post-industrielle qui fait de la consommation une nourriture sans épices et sans âme. L'identité-terminale est ici l'indice probant d'un malaise dans la civilisation. À refuser de marcher, de bouger, le sujet du cocooning est un zombie, un être sans relief. Je le mentionnais un peu plus tôt: survivre n'est pas donné à tous. Qu'on n'y voie pas l'expression facile d'un jeu de mots. Le cocooning révèle une mort lente par asphyxie. La rumeur emprisonne, entoure de toutes parts. Dans cet univers surdimensionné, l'information est un leurre qui permet de conforter l'illusion d'une ambiologie protectrice.

Il en va autrement des sujets de l'exclusion qui habitent des mondes déclassés. Pour survivre, il faut se voir reconnaître la qualité d'«être». Dans l'immense rumeur qui anime l'«Occident» développé, ce sont des pans du monde qui sont oubliés. Dans ces situations, la rumeur s'affaiblit peu à peu. Elle devient atone. Parfois, on entend que «l'Afrique va mal», qu'une «catastrophe humanitaire» a lieu au Darfour. Alors, les écrans des téléviseurs se mettent à scintiller: quelques correspondances de l'étranger dont le montage doit faire dans les soixante secondes imparties, quelques dépêches d'agences de presse, puis le silence. Ce qui devait tenir lieu d'information n'est même pas une rumeur, au plus une plainte sans grande amplitude pour qui contrôle les monopoles de la presse écrite et électronique.

\section{LA GESTION DU PORTEFEUILLE IDENTITAIRE}

Il convient donc de faire preuve d'une prudence absolue à propos des formes actuelles de la délocalisation virtuelle. Il est d'usage d'énoncer que nous vivons dans un monde où il est possible de rompre avec les repères cartographiques, de les intégrer à un imaginaire de synthèse où les vieilles idéologies du sens et de la totalité n'interviennent plus. Ce discours euphorique est tentant, mais je ne le partage pas. Cette idéologie faussaire du jet lag prend l'aspect d'une incartade sans conséquence. Dans cette simulation plus vraie que nature, le jet lag tient lieu de déplacement organisé, de simulation orchestrée. Pas étonnant que le propos de Pico Iyer, auteur et traveler writer fort prisé dans le monde anglo-saxon, propose un itinéraire délocalisé. De Katmandou à Bali, le Global Soul (Pico Iyer) n'est pas autre chose qu'un exotisme virulent. Quant aux sujets de l'exclusion (les anciens mondes "coloniaux" décrits par Leiris et Gide), ils ont laissé place aux espaces-portails, aux «hubs» de la territorialité virtuelle qui circonscrivent un nouveau discours de convivialité identitaire. La lecture des récits de Pico Iyer est pour cette raison éclairante. Elle a le mérite de nous indiquer, mieux que bien des discours universitaires, la très grande facilité avec laquelle nous nous engouffrons dans un monde sans relief, un monde de faux-semblants, une ambiologie conformiste. Pas étonnant que les récits de Pico Iyer se situent en des endroits mythiques (l'Islande, le Népal) et périphériques qui tiennent lieu 
de rumeur exotique. De l'aéroport de Hong Kong au LAX de Los Angeles, c'est la même ritournelle idiote qui prévaut. Les espaces-portails des réseaux de communication nous offrent l'illusion d'une circulation facile, efficace. Le vocabulaire actuel de la marchandisation virtuelle nous impose ce discours.

D’Annie Ernaux à Serge Doubrovsky, la rumeur est en effet devenue un principe fondateur efficace dans la gestion d'un portefeuille identitaire. Comme d'autres manipulent actions et produits dérivés sur le marché boursier, la rumeur est «sous influence». Qu'un Doubrovsky décide brillamment de créer le genre "autofictionnel», il devient le maître de cérémonie d'un art de la présence (et de la disparition) de soi. Alors qu'un Michel Leiris (ou un Sartre avec Les Mots) faisait de la vérité autobiographique un code de l'honneur, la donne autofictionnelle devient une gérance de la rumeur, sa mise sous tutelle. Autrefois, la rumeur avait un «sens»: elle dénonçait secrets, malfaçons et scandales de la vie privée ou sociale. Elle mettait au jour le refoulé. Elle révélait un ordre du monde secret. Il en va autrement aujourd'hui.

Quel intérêt y a-t-il à dévoiler une rumeur déjà connue, scénarisée avec brio par un auteur qui en fait la trame d'un discours autofictionnel? Un Philippe Sollers est sans doute un maitre en ce domaine, tant la frontière entre l'" œuvre» et la «scène» littéraire apparait ténue. Un Jean-Edern Hellier autrefois, un Bernard-Henri Lévy aujourd'hui reprennent à leur compte cette énonciation autocentrée d'un discours où la rumeur est essentielle. Ce n'est pas sans motif que je fais appel à cette image d'une rumeur «sous influence». Dans les pamphlets de Bernard-Henri Lévy, la publication des écrits d'Ernaux et de Doubrovsky, le discours n'a pas pour objet le rétablissement d'une vérité autobiographique. Bien que cet impératif apparaisse toujours dans les écrits de certains de ces auteurs (je pense notamment à Ernaux et à sa valorisation d'une écriture transpersonnelle), il reste que l'enjeu principal n'est pas de dire «vrai», mais de mêler les genres.

À certains égards, l'écriture autofictionnelle devient un prototype de gérance (et de contrôle) de l'image de soi. Sous le prétexte commode d'en finir avec l'injonction dite narcissique du discours autobiographique (Rousseau représentant ici un modèle culturel), cette confusion des genres est une forme actuelle de la rumeur ambiologique. Les mauvais récits autofictionnels (tels ceux de Nelly Arcan) sont des dérivés directs du monde médiatique. Ces publications n'ont de valeur intrinsèque que dans la mesure où ils sont rehaussés par une gérance publicitaire qui ose dire le «vrai», tout en sachant que l'entreprise, par son caractère ostentatoire, renoue avec le monde de la rumeur. Cette gérance de la rumeur n'est à vrai dire pas nouvelle. L'ouvrage d'Edgar Morin, Les Stars, indiquait déjà que le maintien de la célébrité s'appuie sur un «culte» qui donne place à la rumeur. Dans le monde «à part» que constitue l'univers de la star, la rumeur est une caractéristique de cette ambiologie.

Il semble donc que le discours culturel contemporain situe à l'avant-scène ces écotopies ambiologiques qui ont pour fonction première de créer des "climats", des "environnements" sensoriels (tactiles, auditifs, gustatifs) où la question de la «vérité» ne se pose plus. Encore une fois, ce sont les emplacements habités de nos interventions dans le monde qui sont violemment remis en question. Si la vérité n'a plus aucune pertinence (comme enjeu éthique ou pratique dans la construction de nos «arts de faire» quotidiens), c'est qu'elle est devenue un piètre adjuvant dans nos quêtes de fictivité.

Qu'on mesure la radicalité des points de vue en présence: chez les tenants du néolibéralisme cybernétique (pensons à Negroponte, star du magazine Wired et professeur au MIT), la notion de sujet post-humain est une façon de réconcilier les adeptes d'une pure simulation cybernétique de l'activité cognitive et la communauté des «vivants» que nous représentons, sujets faillibles, en proie au doute et à la conscience. Cette «réconciliation" est la fadaise de nombreuses utopies techno-cybernétiques actuelles. Mais il faut aussi prendre garde à ne pas cautionner, avec beaucoup de facilité, un propos frileux et conservateur. 
Il ne suffit pas, comme le fait Pico Iyer, de prendre note des flux de réfugiés qui se multiplient à une vitesse effarante. La prise de conscience ne suffit pas. Elle peut même être d'un insupportable voyeurisme. Les sujets du jet lag et de l'exclusion ne coïncident pas. Ils ne partagent pas les mêmes espaces de sens et de déraison. Le sujet du jet lag est agité. Il est bousculé dans son for intérieur, mais de manière anodine. Le jet lag est un euphorisant mineur. Il permet de dériver dans une béatitude sans effort et sans grande conséquence. D'une certaine manière, le jet lag s'apparente à une toxicomanie bénigne qu'il est possible de convoquer au gré des déplacements. Ce ne sont pas les territoires visités qui importent, mais le rush, le flow, l'extase adrénergique qui sont des conséquences de la fatigue ressentie lors du voyage. Cette drogue douce est notre condition postmoderne. Le jet lag symbolise ce corps refoulé qui ne cesse de baliser nos parcours et nos itinéraires. De façon claire, le jet lag nous indique que le temps du passage - du parcours - est désuet.

\section{UNE AMBIOLOGIE SÉCURITAIRE}

Le jet lag, à sa manière, témoigne de la faillite des espaces publics, de leur transformation en lieux de transit. Du LAX à l'aéroport de Frankfurt, la même dérive - efficace, mécanisée, chorégraphiée - est mise en scène. L'aéroport est devenu un lieu transculturel par excellence: l'hybridité est manifeste, les passagers se croisent sans se parler. Dans une chambre d'hôtel située au Mariott de Montréal ou de Minneapolis, le Welcome et Do Not Disturb des panonceaux (injonctions sémiotiques minimales) ne sont pas là pour vous souhaiter la bienvenue. La chambre d'hôtel postmoderne vous enferme par l'entremise d'une superficialité qui appartient au monde du banal. Cette chambre d'hôtel est un pare-excitations contre un temps-panique, un espace-panique qui pourrait vous mettre à mal. La chambre d'hôtel n'est plus un refuge, un îlot, figures déjà anciennes de l'intimité et du confort. La chambre d'hôtel du Hilton ou du Mariott vous protège des ghettos aux périphéries de l'aéroport. De la même manière, les détenteurs de «cartes privilèges» (Amex, Diners, etc.) dépensent leurs «Air Miles» à proximité de favelas. Cette mise en situation ne correspond pas, de ma part, à une comparaison lyrique qui oppose l'espace fragile des exclus aux lieux impériaux du pouvoir. Cette comparaison est au contraire un acte politiquement informé.

Revenons à cette figure de la chambre d'hôtel du réseau hôtelier international. On peut y voir un espace normé qui nous ramène à la composition disciplinaire des appareils de pouvoir. Fait nouveau, l'ambiologie sécuritaire donne l'apparence d'une banalité qui prévient tout débordement, toute mise en place d'une situation de crise. Ces écotopies ambiologiques font état d'une superficialité explicite. La sérialité est ici le garant d'un retour à l'identique qui n'est pas sans faire penser à la compulsion de répétition freudienne. Pour qui a voyagé, cette sérialité est omniprésente: mêmes réseaux de télévision, même ameublement. Elle est le signe d'une "portabilité» des réseaux écotopiques. Le grand voyageur que décrit Pico Iyer a son «bureau personnel» qui l'accompagne en permanence. $\mathrm{La}$ contemporanéité techniciste et industrieuse s'accompagne de la disponibilité du monde des objets que l'on peut alors convoquer à loisir. Chez Iyer, l'identité-terminale permet d'exercer cette mise en scène de la compulsion de répétition. La sérialité (la banalité répétitive qui fait du monde des objets un ensemble dont on peut permuter les séquences à l'infini) traduit une emprise sur le «réel» qui permet de le réduire à un certain nombre de propriétés formelles. Dans cette mise en ouvre banale et sourde de l'ambiologie, le sujet se doit d'être le maître de l'œkoumène. La manipulation des réseaux cybernétiques à usage personnel rejoint, à bien des égards, cette volonté de domestiquer le monde des objets.

À l'encontre de cette sérialité répétitive de la rumeur marchande, je revendique l'actualité d'un temps-panique qui ne correspond pas au faux catastrophisme dont nous sommes tous les jours informés. Le temps-panique n'est pas la terreur dite terroriste qui fait suite au 11 septembre 2001. Ce temps-panique, s'il a prise sur l'actualité, révèle la 
fragilité des lieux d'être contemporains. À la terreur politique - qui schématise à l'excès celui qui est présenté comme l'ennemi déclaré (de l'Irak aux diasporas arabes en Amérique du Nord) -, il nous faut opposer une lucidité qui fait corps avec la démesure des paysages identitaires contemporains. Il nous faut renouer avec la violence (d'un Genet), la cruauté (d'un Artaud) pour mieux renoncer aux idéologies actuelles du bien commun et de la rectitude morale. Nous sommes encore prisonniers d'une représentation fallacieuse du "beau" et du "bien", alors que nos lieux habités sont des espaces d'enracinement conservateur.

De la chambre d'hôtel anonyme du Mariott à la rumeur politique actuelle (du cyberterrorisme au mouvement Al-Qaïda), nous sommes les personnages d'une ambiologie sécuritaire. L'éloge du cocooning reprend à son compte l'injonction du self-containment, de la censure «intériorisée», de la crainte d'une menace extérieure diffuse et pourtant manifeste. La chambre d'hôtel du Mariott offre de son côté l'image d'une liberté «sous influence». Dans leur niche confortable, le traveler writer (Pico Iyer), le «voyageur de commerce" de la nouvelle économie donnent l'illusion de la mobilité, alors qu'ils vivent dans des espaces contraints. La mobilité actuelle des lieux de séjour est la forme principale d'un arrêt-sur-image, une interruption du temps sur la facticité de l'événement.

Le cocooning est l'incarnation d'un repli sur un monde technique débridé. À distance, le sujet est littéralement nourri d'une somme de stimulations hétéroclites. De son côté, le «jet-setter» est à l'affût de la nouveauté du monde. Mais dans ses pérégrinations, il contemple un monde dont la sérialité est troublante. C'est contre l'image d'une "fausse" mobilité, d'un «faux» déplacement que je situe ma réflexion sur les lieux habités de la rumeur publique. Lorsque je fais référence à ces petites violences traumatiques qui altèrent l'unité de la communauté, la singularité du sujet individuel, j'entends que nous ne sommes pas à l'abri de déflagrations traumatiques. La rumeur publique, tout particulièrement après le 11 septembre 2001, veut absolument nous convaincre que nous sommes menacés et protégés. L'injonction est paradoxale. Elle a pour fonction de nous persuader que nous vivons dans une gigantesque écotopie régressive, sorte de cocooning totalitaire où nous sommes à l'abri du monde tout en contemplant sa mise à mal.

\section{UN MONDE VIOLEMMENT APAISÉ}

De façon paradoxale, l'ambiologie nous offre l'image d'un monde violemment apaisé. C'est la réclame publicitaire qui vous promet l'accès instantané au bonheur de la société de consommation. Mais ce discours vous colle à la peau. Il devient une rumeur qui vous dit de quelle manière vous devez vivre et penser. Il faut bien l'avouer, cette rumeur n'est pas qu'euphorique. Dans les lobby d'hôtels aux États-Unis, les réseaux de télévision Fox News et CNN transmettent une ambiologie sécuritaire qui se répète de demi-heure en demi-heure. Ce discours ambiologique n'est pas différent de la Muzak qui accompagne nos déambulations du restaurant aux ascenseurs des gratte-ciel des grandes villes nordaméricaines. Mais la Muzak, aujourd'hui, c'est le «transtribal», le sampling de la «techno» orchestrée à grande échelle. Ce complément ambiologique est une véritable greffe dont la permanence est la première caractéristique. Dans un aéroport, alors que vous êtes en transit, la diffusion sur écran des informations financières de Bloomberg.com ou de Report on Business s'avère une véritable agression. Mais vous ne pouvez rien y changer. À moins, par exemple, de sortir un «Pod» de votre poche et de choisir, au menu, une symphonie de Bruckner, une plage d'Akufen. Vous aurez alors retrouvé votre liberté en vous isolant, en acceptant le principe monacal d'un repli sur soi. Par votre action, vous renoncerez au Moi-peau ambiologique qui devient un second filtre perceptif.

Voilà pourquoi nous devons interroger le statut de la rumeur ambiologique. Cette ambiologie repose sur l'idée d'environnements sécuritaires et protecteurs où il importe de créer un "climat» sensoriel diffus qui intègre le sujet à un monde perceptif dont il est un 
acteur passif. On objectera qu'il y a peu de différences entre la propagande organisée (outil de coercition et de répression) et l'ambiologie. Y aurait-il d'un côté une argumentation totalitaire, alors que l'ambiologie représenterait, de son côté, le discours d'un libéralisme économique triomphant? J'ai voulu montrer, au cours de cet article, que l'ambiologie caractérise une nouvelle sémiotique de la rumeur. Et j'ai pris le soin d'ajouter que cette sémiotique se caractérise, dans ses règles de fonctionnement, par un littéralisme, une injonction dont l'asémantisme est un trait dominant. Les énoncés: On line, Connect, Delete sont à la fois des énoncés techniques (des codes de conduite sur le réseau) et des modes de représentation du monde qui font appel à l'impartition de l'insourcing et de l'outsourcing. L'ambiologie donne l'illusion du mouvement et de la liberté (d'action), alors que les codes de conduite, les injonctions sont des règles contraignantes qui vous imposent des espaces propres, des écotopies. Ces dernières sont des communautés électives qui possèdent leur propre vocabulaire, leurs règles de cooptation et d'exclusion. Les communautés électives sont littéralistes. Elles font de mots d'ordre d'acceptation et d'appartenance au vivre-ensemble électif la clé d'un «tribalisme» dont il importe de comprendre le caractère dystopique et destructeur.

Le partage d'une ambiologie vous impose de souscrire à des règles d'appartenance. Vous pouvez ainsi appartenir à un réseau si vous vous qualifiez, en somme si vous obéissez à un code commun, à une légitimité partagée. Mais l'ambiologie est à vrai dire plus retorse. Elle ne vous dit pas d'emblée que vous êtes exclu ou coopté. Elle préfère vous laisser le choix d'introjecter un monde binaire: vous pouvez ainsi être un sujet adhérant (suscribe) ou décider de vous soustraire volontairement à une communauté élective (unsuscribe). Dans tous les cas, vous demeurez maître de la situation. Mais l'appartenance à une communauté élective est un leurre. Contrairement à un Michel Maffesoli, qui y voit l'émergence de nouvelles formes festives, mon propos est décidément plus sombre.

Dans la répétition des énoncés asémantiques: Delete, Subscribe, Unsuscribe, nous retrouvons la forme à peine modifiée des exclusions violentes au cœur des ghettos urbains et des territoires occupés. Cette similitude est troublante, tant elle engage une véritable géopolitique du sens qui nous oblige à interroger les rumeurs qui nous accompagnent. Pour cette raison, l'ambiologie est un «climat» perceptif. Avec le concept d'ambiologie, j'ai voulu indiquer que les formes de la persuasion publique connaissent de brutales modifications. Il est en effet trop facile de décréter que l'apparition de nouveaux modes de communication accroît ou interdit de manière systématique la liberté des sujets. Ce discours a été tenu à chaque fois qu'une innovation technique de taille apparaissait. La radio, puis la télévision allaient être de fantastiques outils d'homogénéisation sociale. Ce discours négatif faisait valoir la perte de l'identité locale, singulière. Il en va de même aujourd'hui à propos du monde Internet. À égale distance des thuriféraires du cyberespace et des adversaires de la toile, je souhaite tenir un discours nuancé. Bien évidemment, toute réflexion sur la rumeur est particulièrement adaptée au temps présent.

Dans la rumeur ambiologique, l'intrigue est minimale, la présence d'un énonciateur n'est même plus manifeste. La rumeur est devenue un fait qui correspond aux mondes écotopiques où nous logeons. Marshall McLuhan avançait, il y a plus de trente ans, l'idée d'une géographie culturelle renouvelée qui reposerait sur la puissance organisatrice des réseaux de sens. C'était une intuition profonde dont nous n'avons pas fini de mesurer la portée. Dans la mise en forme actuelle de la rumeur ambiologique, il n'est d'ailleurs plus question de communication, de transmission. La rumeur ambiologique fait corps: elle vous enlace, vous protège; elle vous menace, vous terrorise. Dans l'actualité de la rumeur ambiologique, il y a l'idée d'un «réseau» que l'on peut convoquer à loisir et qui vous fait partager la vie du monde «global». Voilà pourquoi l'homme (et la femme) des quartiers banlieusards redoute d'être seul, et désire à tout prix être «branché». Voilà pourquoi les sans-abris sont les nouveaux exclus de la délocalisation virtuelle. La rumeur ambiologique est devenue notre nouvel espace de sens. Personne n'y échappe. À la manière 
d'un agonisant dont on hésite à «débrancher» l'aide cardio-respiratoire, la rumeur ambiologique est notre nouvelle prothèse. Le sujet postmoderne redoute plus que tout les énoncés d'exclusion: Delete, Reboot. Quant aux sans-logis de la délocalisation virtuelle, ils savent déjà ce qu'est «le voyage au bout de la nuit».

\section{RÉFÉREN CES BIBLIO G RAPH IQ U ES}

AARTS, E. et S. MARZANO [2003]: The New Everyday. Views On Ambiant Intelligence, Rotterdam, 010 Publishers.

AnZIEU, D. [1995]: Le Moi-peau, Paris, Dunod.

BAUDRILLARD, J. [1981]: Simulacres et simulation, Paris, Galilée; [1972]: Pour une critique de l'économie politique du signe, Paris,

Gallimard, coll. «Les essais».

BAUDRILLARD, J. et J. NOUVEL [2000]: Les Objets singuliers: architecture

et philosophie, Paris, Calmann-Lévy.

BENJAMIN, W. [2003]: L'œuvre d'art à l'époque de sa reproductibilité

technique, Paris, Allia.

BION, W. R. [2003]: Aux sources de l'expérience, Paris, PUF, coll.

"Bibliothèque de psychanalyse»;

[1992]: Réflexion faite, Paris, PUF;

- [1979]: Éléments de la psychanalyse, Paris, PUF, coll.

"Bibliothèque de psychanalyse".

CAVELL, R. [2002]: McLuhan in Space. A Cultural Geography, Toronto, University of Toronto Press.

CerTeau, M. de [1990]: L'Invention du quotidien, Paris, Gallimard, coll. «Folio/Essais»;

[1975]: L'Écriture de l'histoire, Paris, Gallimard, coll.

«Bibliothèque des histoires».
Deleuze, G. et F. GuATtARI [1980]: Mille plateaux: Capitalisme et schizophrénie, Paris, Éd. de Minuit.

DURKHeIM, É. [1975]: Religion, morale, anomie, Paris, Éd. de Minuit; [1985]: Les Formes élémentaires de la vie religieuse: le système totémique en Australie, Paris, PUF, coll. "Quadrige».

IYER, P. [2001]: The Global Soul: Jet Lag, Shopping Malls, and the Search for Home, New York, Vintage, 2001;

[1994]: Falling Off the Map, New York, Vintage;

[1989]: Video Night in Kathmandu, New York, Vintage.

LEIRIS, M. [1973]: L'Âge d'homme, précédé de La littérature considérée comme une tauromachie, Paris, Gallimard, coll. «Folio».

MAFFESOLI, M. [1984]: Essais sur la violence banale et fondatrice, Paris, Méridiens.

MCLUHAN, M. [1972]: La Galaxie Gutenberg face à l'ère électronique: les civilisations de l'âge oral à l'imprimerie, Paris, Mame.

Mitchell, W. J. [2000]: Space, Place and the Infobahn. City of Bits, Cambridge (Mass.), MIT Press;

[2003]: The Cyborg Self and the Networked City, Cambridge, MIT Press.

MONs, A. [2002]: La Traversée du visible: images et lieux du contemporain, Paris, Passion

MORIN, E. et B. PAILlaRd [1970] : La Rumeur d'Orléans, édition complétée avec La Rumeur d'Amien, Paris, Seuil, coll. «L'histoire immédiate";

[1984]: Les Stars, Paris, Galilée.

Negroponte, N. [1995]: Being Digital, New York, A. A. Knopf;

[1975]: Soft Architecture Machines, Cambridge (Mass.), MIT Press.

SANSOT, P. [1984]: Poétique de la ville, Paris, Klincksieck, coll.

"Collection d'esthétique";

[1985]: La France sensible, Seyssel (France), Champ Vallon, coll. "Milieux";

[1991]: Les Gens de peu, Paris, PUF, coll. «Sociologie

d'aujourd'hui».

SiMMEL, G. et V. JANKÉLÉVITCH [1993] : La Tragédie de la culture et autres essais, précédé d'un essai de V. Jankélévitch, Paris, Rivages. 